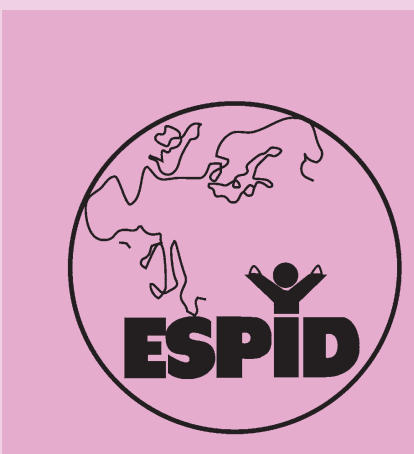

\title{
CONTENTS
}

Point-of-care Testing

EDITORIAL BOARD

Editor: Delane Shingadia

\begin{tabular}{lll} 
& \multicolumn{2}{c}{ Board Members } \\
David Burgner (Melbourne, & Cristiana Nascimento-Carvalho & $\begin{array}{l}\text { George Syrogiannopoulos } \\
\text { Australia) }\end{array}$ \\
Kow-Tong Chen (Tainan,Taiwan) & (Bahia, Brazil) & (Laille Peltola (Turku, Freece) \\
Luisa Galli (Florence, Italy) & Emmanuel Roilides (Thessaloniki, & Tobias Tenenbaum (Mannhein, Germany) \\
Steve Graham (Melbourne, & Greece) & Marceline Tutu van Furth (Amsterdam, \\
Australia) & Ira Shah (Mumbai, India) & The Netherlands)
\end{tabular}

\section{Point-of-care Testing in Pediatric Infectious Diseases}

\author{
Kristina Keitel, PhD, MD, * Laurence Lacroix, MD, $†$ and Alain Gervaix, MD $†$
}

$A^{p}$ point-of-care test is broadly defined as diagnostic test that is performed at or near the site of care. It has a fast turnaround time enabling an expedited clinical decision that may lead to an improved patient outcome. Generally speaking, point-of-care tests for pediatric infectious diseases can be classified into 2 categories: pathogen-specific tests (eg, respiratory syncytial virus) or host biomarkers (eg, C-reactive protein [CRP], procalcitonin [PCT]). The number of available tests has increased exponentially, also fueled by the high demand in resource-limited settings for point-of-care solutions where laboratory infrastructures are lacking (eg, HIV and malaria). However, many unmet needs remain either for specific pathogens or because tests only partially meet the basic requirements for an implementable test that may lead to an improvement in patient outcomes. We summarize the requirements for a high-impact point-of-care test for pediatric infections.

\section{Accepted for publication September 7, 2017.}

From the *Pediatric Emergency Department, Bern University Hospital, Bern, Switzerland; and $\uparrow$ Pediatric Emergency Division, Department of Child and Adolescent, Geneva University Hospitals, Geneva, Switzerland.

The authors have no funding or conflicts of interest to disclose.

Address for correspondence: Alain Gervaix, MD, Service d'Accueil et d'Urgences Pédiatriques, Département de l'Enfant et de l'Adolescent, Hôpitaux Universitaires de Genève, Rue Willy Donzé 6, 1205 Genève, Switzerland. E-mail: alain.gervaix@ hcuge.ch.

Copyright ( 2017 Wolters Kluwer Health, Inc. All rights reserved.

ISSN: 0891-3668/18/3701-0108

DOI: $10.1097 /$ INF.0000000000001806

\section{CRITERIA FOR AN IDEAL POINT-OF-CARE TEST}

For a test to allow expedited diagnosis and an improvement in health outcome, several criteria have to be met. The World Health Organization Sexually Transmitted Diseases Diagnostics Initiative developed the "ASSURED" criteria detailing benchmark characteristics for a point-of-care test in resource-limited settings. ${ }^{1}$ Many of the criteria can be expanded to point-of-care tests in pediatric infectious diseases more broadly. We propose the following criteria (Table 1) based on which point-of-care tests should be rigorously assessed before implementation in clinical practice.

\section{Cost-effectiveness}

Affordability is driven heavily by the context of implementation, and cost-effectiveness can be difficult to define because benefits are often indirect and long term, for example, the mitigation of antibiotic resistance through the use of point-of-care biomarkers to improve antibiotic prescription. Nevertheless, costing data should be collected prospectively in research studies for cost-benefit analysis to allow stakeholders to make informed policy decisions.

\section{Sufficient Diagnostic Accuracy and Reliability}

An important first step in a test's validation is the determination of its accuracy and reliability. Such studies have been performed for most commercially available point-of-care tests, usually through estimation against a diagnostic gold standard. Several issues should be considered when performing accuracy studies.
First, adequate and comparable gold standards are often not available for pediatric infections. This is because microbiologic laboratory testing is neither perfectly sensitive nor specific and cannot differentiate between true infection (causing the present infectious episode), comorbid infection, incidental infection and carrier state. Imaging studies also have limitations: for example, consolidation on chest radiograph has been used as a surrogate for bacterial pneumonia, but only a minority of radiographic pneumonia is bacterial in origin. ${ }^{2}$ Furthermore, gold standards may be defined variably across research groups: for example, some consider pyuria as a diagnostic criterion for urinary tract infection in addition to bacteriuria, while others do not.

Second, accuracy studies should be performed in the context in which the test will be used. The performance characteristics of a test will depend on the epidemiologic context and level of care (spectrum bias). Most host biomarker validation studies have been carried out in hospital settings. ${ }^{3}$ Findings may not be directly translatable to lower prevalence, outpatient settings. In general, the aim of performing point-of-care tests for pediatric infections is usually not to categorically make or exclude a diagnosis but to generate a posttest probability through interpretation of the test result based on the pretest probability. Point-of-care tests are not suitable as screening tests but should be applied only to patients with sufficient pretest probability of having the disease. As a result, the assessment of the accuracy of any diagnostic test should be performed within specific clinical scenarios and with the future, specific purpose of the diagnostic test in mind. ${ }^{4}$

The ESPID Reports and Reviews of Pediatric Infectious Disease Journal series topics, authors and contents are chosen and approved independently by the Editorial Board of ESPID. 
TABLE 1. Quality Criteria for Point-of-care Test

$\begin{array}{ll}\begin{array}{l}\text { World Health Organization } \\ \text { ASSURED Criteria }\end{array} & \begin{array}{r}\text { Proposed Criteria for an Ideal Diagnostic Point-of-care } \\ \text { Test in Pediatric Infectious Diseases }\end{array} \\ \begin{array}{l}\text { Affordable } \\ \text { Sensitive } \\ \text { Specific }\end{array} & \begin{array}{l}\text { Cost-effective } \\ \text { Sufficient diagnostic accuracy and reliability }\end{array} \\ \text { User-friendly } & \text { Impact on patient outcome/clinical benefit } \\ \text { Robust and rapid } & \text { User-friendly } \\ \text { Equipment-free } & \begin{array}{l}\text { Rapid } \\ \text { Deliverable to those who need them }\end{array}\end{array}$

The use of a combination of several tests, either concomitantly or sequentially, has become an attractive prospect for pointof-care testing, for example, using several host biomarkers to identify children with serious bacterial infection ${ }^{5}$ or pathogenspecific multiplex platforms after an initial biomarker rule-in step. Combinations of tests should be evaluated in diagnostic accuracy studies. There is a risk that using multiplex solutions may lead to undesired "side effects" in that pathogens are identified that would have usually not been searched for by the clinician, which may lead to unnecessary antibiotic treatment. Finally, test performance characteristics may vary by site of operation. Results from laboratory-based studies may differ from findings when a test is operated in a clinical setting. ${ }^{4}$ Ideally, a test should hence be validated at the targeted site of operation.

\section{Impact on Patient Outcome}

In addition to the assessment of analytical performance, validation studies should assess the impact on relevant patient outcomes. Such outcomes, among others, include mortality, complications from infections, hospitalizations, duration of hospitalization and rates of antibiotic prescription. In pediatric infectious diseases, the vast majority of studies have focused on test accuracy assessments and only few measured the effect of point-of-care testing on patient outcomes in the appropriate clinical setting. ${ }^{6,7}$ Outcome-based evaluations are of particular importance for pediatric infections due to the abovementioned lack of adequate gold standards. As a consequence, missing a diagnosis based on available surrogate markers (eg, radiographic consolidation as an imperfect marker for bacterial pneumonia) may not translate into clinically relevant patient outcomes (eg, complications if no antibiotic treatment is given). Conversely, a test with only moderate performance in accuracy studies may actually have a significant impact on patient outcomes. For example, initiating a treatment early based on a rapid point-of-care test may provide advantages over delaying treatment for a more accurate, laboratory-based test result (eg, antibiotic treatment for multidrug resistant tuberculosis based on rapid "Xpert MTB/RIF" testing rather than culture and drug sensitivity testing).

\section{User-friendly, Rapid and Adequate Technology}

Though many novel host biomarkers and pathogen-specific tests have been developed, few are available in point-of-care formats. The efficiency of any test, or combination of tests will depend on many operational considerations and having an adequate test platform will be a key to impact on health outcomes. ${ }^{4}$ Many types of technology and methods of use have been developed and can translate into a point-of-care test. ${ }^{8}$ The following basic requirements should be considered:

The requirements for a testing platform depend above all on the patient population and care setting. Point-of-care tests in pediatrics are most often applied in outpatient settings and as the type of sample plays a particular role, collection should be simple. Saliva (not sputum), nasopharyngeal swabs and capillary blood are the easiest to obtain. Urine is acceptable but difficult to collect sterilely in pediatric patients. For application in resource-limited settings, a test platform has to be robust to meet further requisites such as nonreliance on electricity, running water or refrigeration, resistance to heat and dust, availability and storage of reagents. A platform should be able to run several test applications. The ideal format would be an open-source solution into which numerous tests can be built in. This would avoid the requirement for several test platforms, limited operational errors, simplified training and quality management needs, and streamline data connectivity issues. Data management systems should be vendor-neutral and compatible across different platforms. This will also allow the integration of point-of-care test results with electronic health systems.

Platforms should be easy to operate to minimize errors, as they are handled by staff with very limited training in laboratory procedures. For example, testing should be as automated as possible and not involve further sampling processing steps (such as centrifugation or pipetting). The turnaround time of a test should be fast enough to expedite clinical decision-making, ideally less than 15 minutes. As selection of appropriate candidates for testing and the interpretation of test results in the epidemiologic context are important, the application of point-ofcare tests by providers with limited clinical training requires clear guidance and clinical algorithms for appropriate and optimal usage.

\section{CRP AND PCT: ESTABLISHED POINT-OF-CARE BIOMARKERS FOR PEDIATRIC INFECTIONS}

CRP and PCT have been assessed comprehensively for the performance in detecting children with serious bacterial infections. Van den Bruel et $\mathrm{al}^{3}$ systematically reviewed diagnostic accuracy studies. Both CRP and PCT had diagnostic value in ruling in and ruling out serious bacterial infections among pediatric patients presenting with fever (positive likelihood ratios ranged 2.4-3.8 for CRP, 1.8-3.1 for PCT; negative likelihood ratios ranged $0.3-0.6$ for CRP, $0.1-0.4$ for PCT). Both tests outperformed white blood cell count. Several studies have assessed the diagnostic accuracy of PCT and CRP for the diagnosis of pyelonephritis using dimercaptosuccinic acid scan as a gold standard diagnosis. A recent Cochrane review reported that PCT at a cutoff of $0.5 \mathrm{ng} / \mathrm{mL}$, and CRP at a cutoff of $20 \mathrm{mg} / \mathrm{L}$ had a sensitivity of $86 \%$ and $94 \%$ and a specificity of $74 \%$ and $39 \%$, respectively. ${ }^{9}$ There is also evidence that CRP and PCT are helpful in detecting bacterial pneumonia in children. In the recent Pneumonia Etiology Research for Child Health Study, a CRP cutoff of $37 \mathrm{mg} / \mathrm{L}$ distinguished 119 HIV-negative children with bacterial pneumonia from 556 HIV-negative children with respiratory syncytial virus pneumonia with a sensitivity of $77 \%$ (95\% confidence interval [CI]: $69 \%-84 \%$ ) and a specificity of $82 \%$ (95\% CI: $78 \%-85 \%)$. Similarly, CRP and PCT were helpful in detecting children with radiographic consolidation suggestive of bacterial pneumonia in a Swiss study of 142 children with pneumonia. ${ }^{10}$ One major limitation of CRP and PCT is that they are both markers targeted for bacterial infection. Recent evidence has suggested that combining bacterial markers with viral markers of infection, such as the combination of CRP, TRAIL and IP10, may yield better diagnostics performance. ${ }^{11}$ The performance of this combination in outcome-based studies remains to be determined and point-of-care test solutions are still under development. 


\section{CONCLUSIONS}

Point-of-care tests for the diagnosis of infections among pediatric patients are increasingly available with the potential to improve patient outcomes as they may allow expedited clinical decision-making. Their potential impact is especially high in resource-limited settings where laboratory infrastructures are lacking. Any point-of-care test should be rigorously assessed on the basis of test accuracy and impact on patient outcome at the intended site of use before routine implementation. Clinical decision algorithms should be developed and validated that allow appropriate selection of patients for testing and the correct interpretation of test results. The efficiency of any test will depend on additional operational variables such as cost and adequate testing platforms, which should be considered early on. A multidisciplinary approach is required to develop effective and applicable point-of-care diagnostic solutions to improve outcomes of pediatric patients with infections.

\section{REFERENCES}

1. Peeling RW, Holmes KK, Mabey D, Ronald A. Rapid tests for sexually transmitted infections (STIs): the way forward. Sex Transm Infect. 2006;82:v1-v6. doi:10.1136/sti.2006. 024265 .

2. Lynch T, Bialy L, Kellner JD, et al. A systematic review on the diagnosis of pediatric bacterial pneumonia: when gold is bronze. PLoS One. 2010;5:e11989.

3. Van den Bruel A, Thompson MJ, Haj-Hassan T, et al. Diagnostic value of laboratory tests in identifying serious infections in febrile children: systematic review. BMJ. 2011;342:d3082.

4. Drain PK, Hyle EP, Noubary F, et al. Diagnostic point-of-care tests in resource-limited settings. Lancet Infect Dis. 2014;14:239-249.

5. Kapasi AJ, Dittrich S, González IJ, et al. Host biomarkers for distinguishing bacterial from non-bacterial causes of acute febrile illness: a comprehensive review. PLoS One. 2016;11:e0160278.
6. Lacroix L, Manzano S, Vandertuin L, et al. Impact of the lab-score on antibiotic prescription rate in children with fever without source: a randomized controlled trial. PLoS One. 2014;9:e115061.

7. Verbakel JY, Lemiengre MB, De Burghgraeve T, et al. Should all acutely ill children in primary care be tested with point-of-care CRP: a cluster randomised trial. BMC Med. 2016;14:131.

8. St John A, Price CP. Existing and emerging technologies for point-of-care Testing. Clin Biochem Rev. 2014;35:155-167.

9. Shaikh N, Borrell JL, Evron J, et al. Procalcitonin, C-reactive protein, and erythrocyte sedimentation rate for the diagnosis of acute pyelonephritis in children. Cochrane Database Syst Rev. 2015;1:CD009185.

10. Alcoba G, Keitel K, Maspoli V, et al. Three-step diagnosis of pediatric pneumonia at the Emergency department using clinical predictors, C-reactive protein, or procalcitonin, and pneumococcal or viral PCR. Eur Jounal Pediatr. 2017;176(6):815824. doi:10.1007/s00431-017-2913-0.

11. Srugo I, Klein-Kremer A, Stein $M$, et al. Validation of a novel assay to distinguish bacterial and viral infections. Pediatrics. 2017;140. pii: e20163453. 